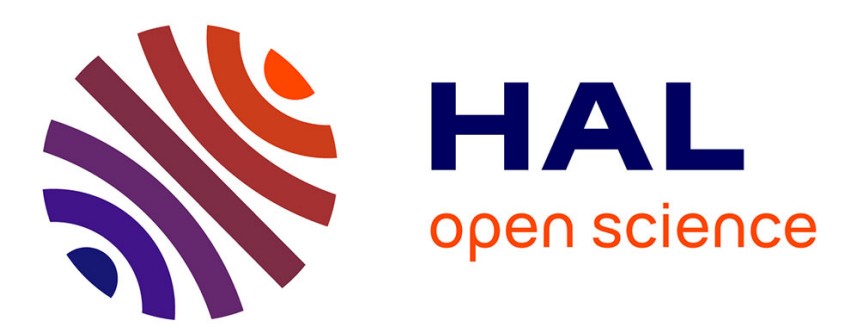

\title{
Système d'acquisition, visualisation, dépouillement en ligne d'expériences simultanées
}

\author{
J.C. Brun, L. Faucher, J.P. Adam
}

\section{To cite this version:}

J.C. Brun, L. Faucher, J.P. Adam. Système d'acquisition, visualisation, dépouillement en ligne d'expériences simultanées. Revue de Physique Appliquée, 1969, 4 (2), pp.147-148. 10.1051/rphysap:0196900402014700 . jpa-00243188

\section{HAL Id: jpa-00243188 \\ https://hal.science/jpa-00243188}

Submitted on 1 Jan 1969

HAL is a multi-disciplinary open access archive for the deposit and dissemination of scientific research documents, whether they are published or not. The documents may come from teaching and research institutions in France or abroad, or from public or private research centers.
L'archive ouverte pluridisciplinaire HAL, est destinée au dépôt et à la diffusion de documents scientifiques de niveau recherche, publiés ou non, émanant des établissements d'enseignement et de recherche français ou étrangers, des laboratoires publics ou privés. 


\title{
SYSTÈME D'ACQUISITION, VISUALISATION, DÉPOUILLEMENT EN LIGNE D'EXPÉRIENGES SIMULTANÉES
}

\author{
Par J. C. BRUN, L. FAUCHER et J. P. ADAM (1), \\ Institut de Physique Nucléaire, 9I-Orsay.
}

\begin{abstract}
Résumé. - Le système installé à Orsay est conçu pour permettre la gestion simultanée de plusieurs expériences de physique se déroulant de façon indépendante dans plusieurs salles. Une voie d'acquisition par utilisateur est connectable sur tout l'appareillage classique de physique nucléaire. Les postes à distance sont composés d'une machine à écrire, d'une unité de visualisation (télévision) associée à un crayon photosensible (light-pen).
\end{abstract}

Abstract. - The computer system set up at Orsay is designed to control simultaneously several independent experiments made in different places. An acquisition channel can be conventional nuclear physics equipment. The remote stations are equipped with a type writer, a visual display (television), an associated light-pen.

Le système installé à Orsay est conçu pour la gestion simultanée de plusieurs expériences de physique nucléaire se déroulant de façon indépendante dans plusieurs salles situées de 400 à $800 \mathrm{~m}$ du centre de traitement. La caractéristique la plus importante des conditions d'exploitation est la grande variété d'expériences nécessitant des programmes différents : a) identification de particules $E, \Delta E / \Delta x ; b)$ identification de particules par localisation après un analyseur magnétique : $(E, p)$, chambre à fils, etc.; c) corrélation angulaire $\left(E_{1}, E_{2}, \theta\right)$; d) spectroscopie avec histogrammes de grandes dimensions : $1024 \times 1024$ canaux, etc. Techniquement, le système doit pouvoir acquérir de l'informatıon codée à partir des convertisseurs existant dans le laboratoire (CA 25, CA 13, Intertechnique, Laben, SAIP). Il faut aussi recopier des groupes de comptage. L'organisation actuelle de l'appareillage (Hardware) du système est représentée sur la figure 1. Signalons que l'exploitation à distance du réseau de BM 96 est actuellement à l'étude.

I. Programmation. - Le système est utilisé pour gérer des expériences en ligne, dépouiller en mode connecté, dépouiller hors ligne et mettre au point les programmes des classes précédentes. Nous avons adopté la ligne de conduite suivante : 1) utiliser le plus possible les ressources offertes par le système d'exploitation standard du fabricant de calculateur; 2) imposer le moins de restrictions possible à la logique et à l'organisation des programmes en ligne qui doivent pouvoir être écrits et modifiés rapidement; 3 ) dégager le physicien du souci de gérer les ressources de base du

(1) IBII France.

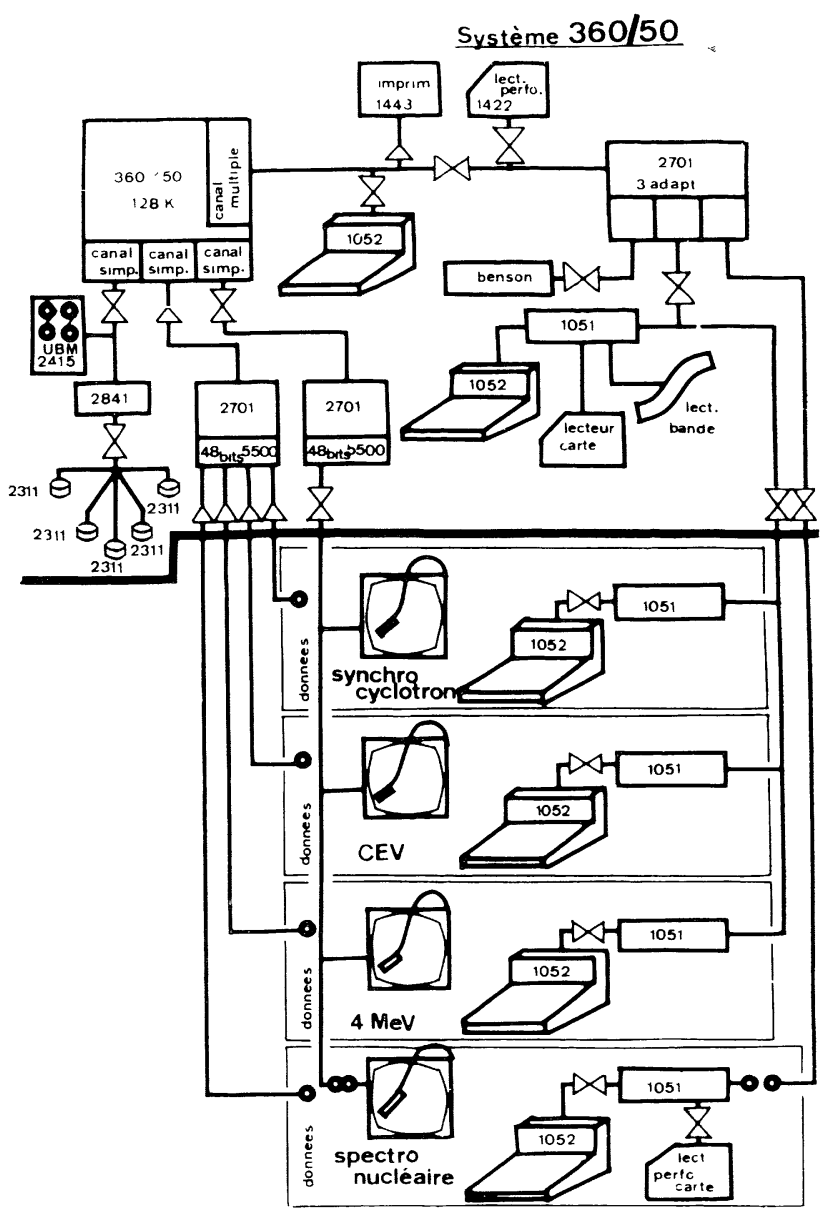

FIG. 1. - Diagramme général. 


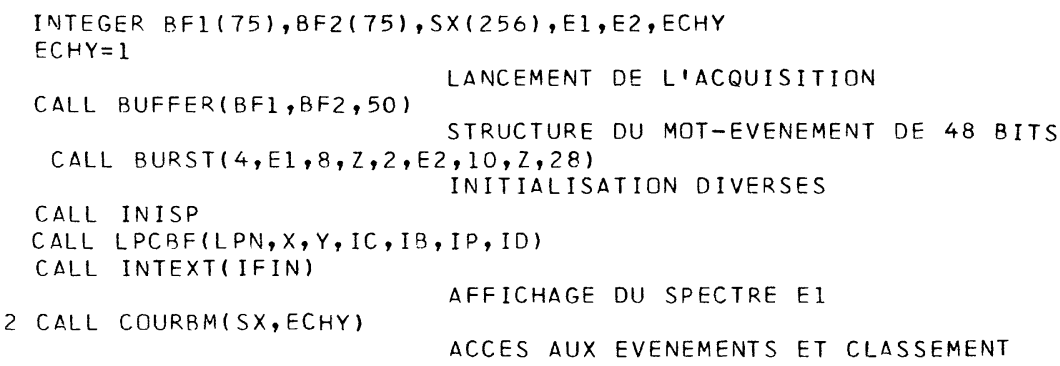

IG. 2. - Exemple de programme : Ce programme constitue en temps réel un spectre bi-dimensionnel de $256 \times 1024$ canaux sur disque, affiche le spectre sur E1 et effffectue les modifications d'échelle sous le contrôle du light-pen.

calculateur. Nous utilisons largement le « Fortran »; 4) permettre la gestion simultanée de plusieurs expériences. Cela recouvre des modifications du superviseur IBM (actuellement Disk Operating System) en y insérant les organes d'acquisition et de visualisation.

Les programmes des physiciens se présentent donc sous forme relativement simple (exemple de programme d'acquisition à 256000 canaux avec contrôle par visualisation ( fig. 2)).

II. Visualisation. - Les caractéristiques en sont les suivantes : a) visualisation à trame genre télévision 625 lignes (verticales), 50 images $/ \mathrm{s} ; b$ ) régénération de l'image en mémoire centrale (utilisation des possibilités de simultanéité du canal simple 360/50); c) association d'une machine à écrire; d) branchement d'un light-pen à distance.

Au début de l'année 1969, nous mettrons en service : un générateur de caractères alpha numérique (256 caractères sur l'écran), 48 symboles, et une extension des possibilités des postes de visualisation permettra de présenter 128000 points sur l'écran.

Les programmes de base sont actuellement entièrement supportés par le superviseur qui gère la simultanéité des postes et des light-pen.

Nous disposons d'un premier niveau de fonctions accessibles sous forme de call Fortran permettant d'appeler des courbes, de définir l'état des points pré. sentés (normaux, brillants, clignotants, etc.), de prendre en considération l'information light-pen, etc. Un second niveau des programmes recouvre des fonctions plus générales de visualisation (échelle, centrage, exploration d'un volume, intégration, etc.). La conversation homme-machine ne recouvre que l'adaptation correcte des programmes aux besoins (converser avec un calculateur signifie seulement brancher sur ordre extérieur le programme dans une direction souhaitée).

Nous avons étudié par ailleurs des programmes importants de dépouillement par visualisation, par exemple : a) exploration de spectres de très grande dimension $\left(10^{6}\right.$ canaux $)$ résidant sur disque; $b$ ) séparation de masse sur visualisation en utilisant simultanément des images de courbe de niveau ou de coupes isométriques et des images de section. Nous comptons réaliser un programme de séparation $E, \Delta E / \Delta x$ permettant d'extraire une masse au light-pen. Cette méthode « topologique » de séparation diffère très sensiblement des méthodes de calcul. Nous pensons que la séparation définitive (optimale) prendra au plus une demi-heure de travail de physicien pour chaque masse, et ceci immédiatement après l'acquisition.

III. Conclusion. - L'expérience de six mois d'exploitation nous a montré les possibilités de l'outil qu'est ce système. 